\title{
Erratum to: Clinical and molecular genetics of neonatal diabetes due to mutations in the insulin gene
}

\author{
Julie Støy • Donald F. Steiner • Soo-Young Park • \\ Honggang Ye • Louis H. Philipson • Graeme I. Bell
}

Published online: 1 February 2011

(C) Springer Science+Business Media, LLC 2011

Erratum to: Rev Endocr Metab Disord (2010) 11:205-215

DOI 10.1007/s11154-010-9151-3

The following INS gene mutation was numbered incorrectly: In Figure 1, Figure 2, and section 6.1, we refer to the mutation V93L. This should have read as V92L. This does not affect the numbering of the other mutations, which remain correct. The corrected versions of Figures 1 and 2 are presented below. We apologize for this error.

The online version of the original article can be found at http://dx.doi. org/10.1007/s11154-010-9151-3.

J. Støy $(\bowtie)$

Department of Internal Medicine and Endocrinology,

Aarhus University Hospital,

Nørrebrogade 44,

8000, Aarhus C, Denmark

e-mail: julistoe@rm.dk

D. F. Steiner $\cdot$ S.-Y. Park $\cdot$ H. Ye $\cdot$ L. H. Philipson $\cdot$ G. I. Bell

Department of Medicine, Human Genetics,

and Biochemistry and Molecular Biology,

The University of Chicago,

5841 S. Maryland Ave., MC1027,

Chicago, IL 60637, USA 


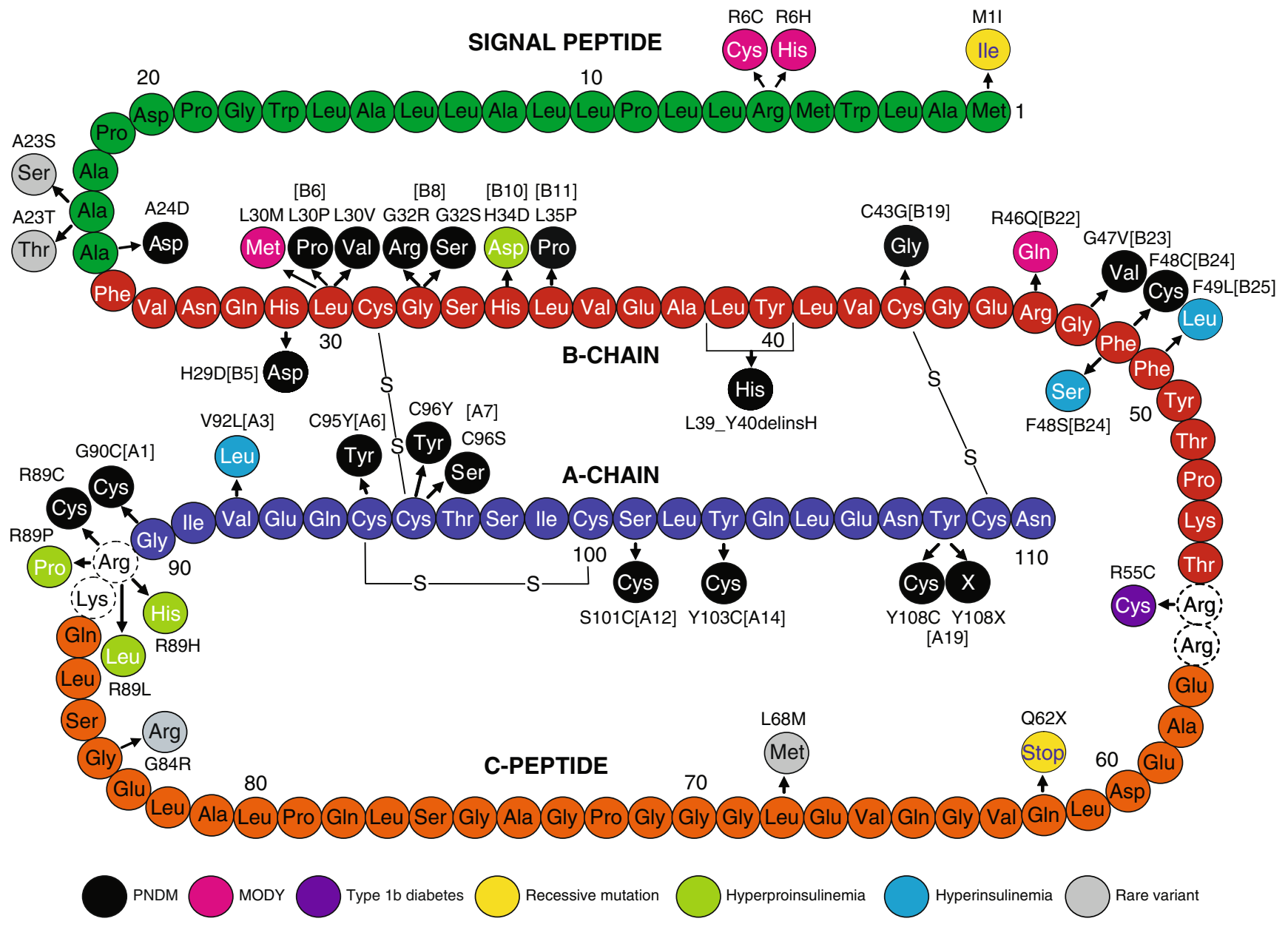

Fig. 1 Diagrammatic representation of the amino acid sequence of human preproinsulin (signal peptide-green, B-chain-red, C-peptideorange, A-chain-dark blue) indicating sites of mutations identified in patients with diabetes as well as hyperinsulinemia and hyperproinsulinemia. Mutations shown in black disrupt proinsulin folding and/or disulfide bond formation leading to permanent neonatal diabetesmellitus (PNDM); mutations in light blue do not impair folding but are associated with reduced insulin receptor binding potency (hyperinsulinaemia); mutations in light green are associated with hyperproinsulinaemia and either impair proteolytic processing to insulin or, in the case of H34D, aggregation and sorting into dense-core granules of the regulated secretory pathway. Mutations in pink and purple were found in patients with a diagnosis of maturity-onset diabetes of the young (MODY) and type $1 \mathrm{~b}$ diabetes, respectively. The $\mathrm{R} 55 \mathrm{C}$ mutation has been found in patients with a diagnosis of type $1 \mathrm{~b}$ diabetes as well as MODY. The mutations shown in gray (A23S, A23T, L68M and G84R) are rare variants without functional effects on proinsulin/insulin biosynthesis. The mutations shown in yellow are recessive mutations that affect insulin biosynthesis (mutation of the translation initiation Met) or cause the synthesis of a nonsense protein (Q62X) 


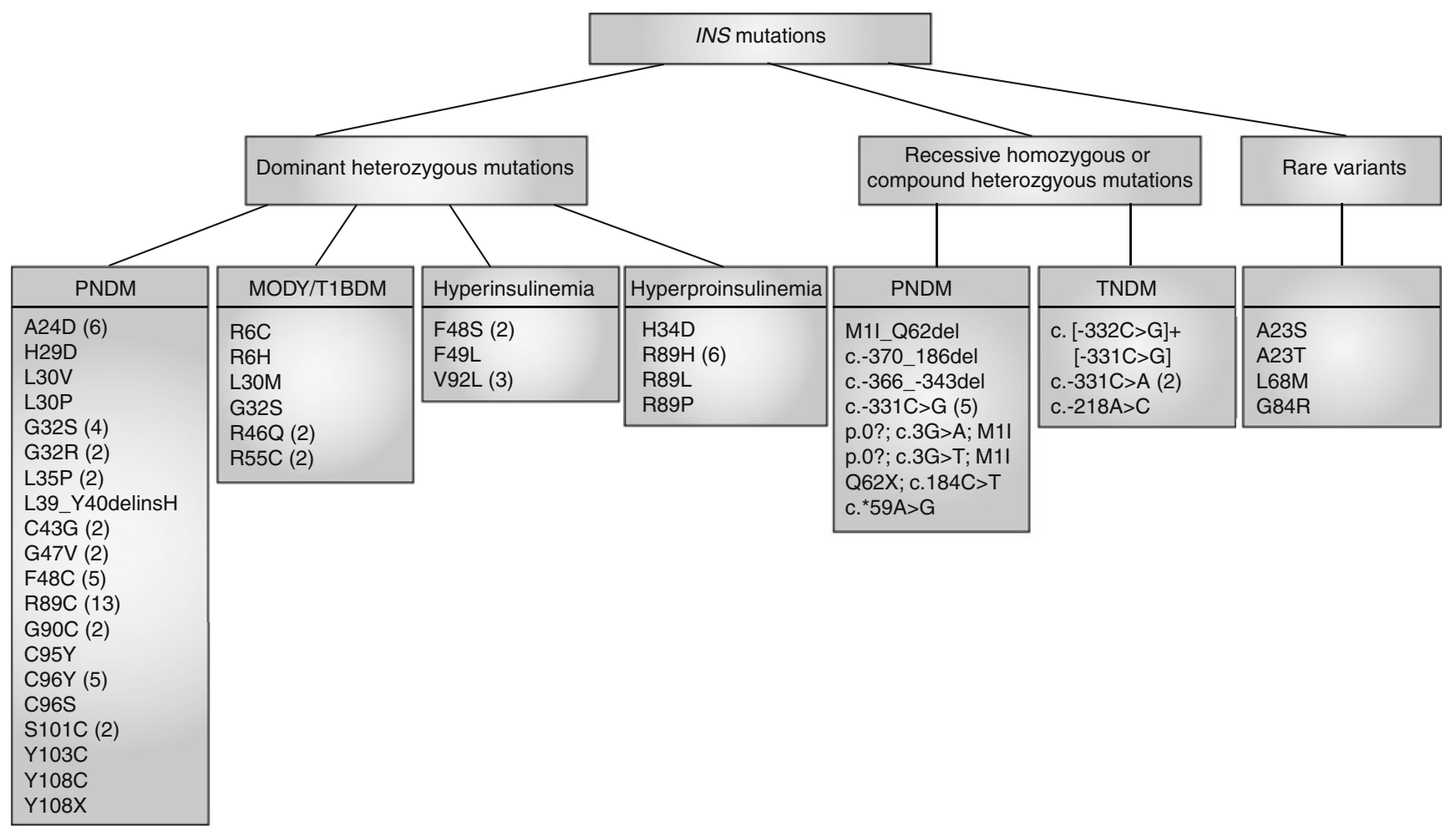

Fig. 2 Summary of human insulin gene mutations and disease phenotype. The numbers in brackets indicate the number of probands with that specific mutation. PNDM, permanent neonatal diabetes mellitus; TNDM, transient neonatal diabetes mellitus; MODY, maturity-onset diabetes of the young; T1BDM, type 1b diabetes mellitus 